\title{
Research Progress of Lower Limb Rehabilitation Robots in Mainland China
}

\author{
Zheng Liu, Dunmin Lu* \\ School of Technology, Beijing Forestry University, Beijing, China \\ Email: *dunmin_lu@bjfu.edu.cn
}

How to cite this paper: Liu, Z. and $\mathrm{Lu}$, D.M. (2019) Research Progress of Lower Limb Rehabilitation Robots in Mainland China. Open Journal of Therapy and Rehabilitation, 7, 92-105. https://doi.org/10.4236/ojtr.2019.73006

Received: May 29, 2019

Accepted: June 17, 2019

Published: June 20, 2019

Copyright $\odot 2019$ by author(s) and Scientific Research Publishing Inc. This work is licensed under the Creative Commons Attribution International License (CC BY 4.0).

http://creativecommons.org/licenses/by/4.0/

\section{(c) (i) Open Access}

\begin{abstract}
The number of people with lower limb disabilities caused by stroke, traffic accidents and work-related injuries is increasing sharply every year in mainland China, and the corresponding number of rehabilitation therapists is obviously insufficient. To solve this problem, domestic large hospitals have introduced advanced lower limb rehabilitation robots from abroad. However, such robots are expensive and the number of them cannot meet the needs of patients. As a result, many universities and colleges in mainland China have launched research on this issue. This paper collects and collates the research literature, gives the mature and typical structure and control system design scheme in mainland China, and lists some representative research results. Finally, the rehabilitation effect of these lower limb rehabilitation robots is evaluated.
\end{abstract}

\section{Keywords}

Lower Limb Rehabilitation Robots, Research Progress, Mainland China

\section{Introduction}

Aiming at the lower limb disability caused by various reasons such as stroke, aging, traffic accidents, etc., the long-term lack of effective exercise of the limbs of patients will lead to the reduction of blood circulation in joints and muscles, the failure of blood to flow smoothly in tissues, and the decline of body resistance, and then lead to various diseases, such as muscle atrophy, ligament shortening, joint stiffness, or abnormal motion patterns [1] [2]. Medical theory and clinical medicine have proved that for the limb disabled, on the one hand, it is necessary to receive pre-operative treatment, just like that drug treatment, on the other hand, still needs to use scientific limb training methods in order to better restore the patient's limbs to a better state. Therefore, correct and scien- 
tific rehabilitation training plays a very important role in the recovery and improvement of limb motor function [3].

At present, the traditional artificial rehabilitation therapy is mostly used in clinic. This method consumes a lot of manpower, material resources, financial resources and time, which is far from meeting the needs of most patients for rehabilitation treatment. Therefore, there is an urgent need for a treatment method that can replace traditional rehabilitation therapists.

Rehabilitation engineering is an integrated field of rehabilitation medicine and robotics technology. By applying advanced robotics technology to rehabilitation medicine, it can provide patients with assistant training or treatment equipment. Rehabilitation robot is a comprehensive product applied in rehabilitation engineering. Its research integrates many fields such as robotics, rehabilitation medicine, ergonomics, computer science, and so on. It is a hot research direction in the field of international robotics technology and has developed into an important component of medical robots. Rehabilitation robots can be divided into two types according to their functions: rehabilitation training type and rehabilitation assistant type. At present, rehabilitation robot technology has been widely used in rehabilitation treatment and rehabilitation nursing.

At present, most of the major hospitals in mainland China adopt lower limb rehabilitation robots abroad, such as Lokomat [4] [5] in Switzerland and HAL [6] [7] in Japan. They are sophisticated in technology, but expensive at the same time. In order to solve this problem, domestic universities have begun to study. Among them, the representative ones are Harbin Engineering University, Yanshan University, Shanghai Jiao Tong University and so on.

This paper collects and analyses the increasing domestic research literature on lower limb rehabilitation robots. The second chapter mainly discusses the structural design of the existing lower limb rehabilitation robot in mainland China. In the third chapter, the design scheme of the control system is studied. The fourth chapter gives some representative examples of lower limb rehabilitation robots in mainland China. Then the fifth chapter evaluates their rehabilitation effect. Finally, the prospects for the development of lower limb rehabilitation robots in mainland China are prospected.

\section{Structural Design of Lower Limb Rehabilitation Robot}

The lower limb rehabilitation robot mainly includes assistant training mechanism and control systems.

\subsection{Design Requirements of Lower Limb Rehabilitation Robot}

As one of the rehabilitation equipment, the lower limb rehabilitation robot needs to meet the following requirements:

1) Safety: Because lower limbs of hemiplegia and paraplegia patients caused by stroke and other diseases lack effective movement for a long time, muscles atrophy, ligament shortening, joint stiffness or abnormal motion patterns appear. 
Therefore, the safety of the robot must be guaranteed first in the structural design. It includes the selection of appropriate materials to ensure structural strength, reasonable structural design to ensure stiffness, as well as with limit switches, emergency stop buttons and other safeguards.

2) Effectiveness: Lower limb rehabilitation robot should meet the rehabilitation needs of patients, so that lower limbs of patients can be fully exercised and muscle strength can be restored. Ultimately, it should be able to help patients achieve the functions of standing, walking and so on.

3) Universality: As a commodity, lower limb rehabilitation robot should be able to meet the needs of various users. It can be adjusted accordingly to maintain comfort for patients with different body shapes.

4) Economy: The purpose of domestic research on lower limb rehabilitation robot is to alleviate the shortage of supply in the domestic market, and ultimately make its price in a reasonable range. At the same time, the robot should be made as beautiful and compact as possible.

\subsection{Exoskeleton Structure Design}

In mechanism design, the training of hip, knee and ankle joints of both lower limbs should be realized. Based on the actual rehabilitation process of hemiplegia, two kinds of training methods, joint separation and joint compound exercise, can be provided to meet the needs of different rehabilitation stages. We should try our best to make the joint separation training of the robot meet the training requirements of range of motion of the single joint of patients, realize the whole range of motion of the single joint, and maintain the range of motion of the joint. When spasm occurs, joint separation training can be used for intensive training of spastic joints. Compound joint training is a comprehensive training for lower limbs. It not only completes the training trajectory planned by rehabilitation therapists according to the patient's condition, but also hopes to simulate walking training and reconstruct the normal walking pattern.

According to the needs of general rehabilitation training, three degrees of freedom are allocated to the hip joint, one degree of freedom to the knee joint and two degrees of freedom to the ankle joint. There are two methods to design joint drive mechanism. One is driven by DC servo motor with screw-nut transmission, the other is driven directly by motor. Among them, the former is mostly used because it can provide larger torque. Limit switches are also needed to prevent joint torsion from exceeding the limit of human activity. Length of thigh and calf should be adjusted to meet the needs of patients with different height.

Because the standing and walking function of lower limbs of patients is partially or completely lost, it is difficult to achieve standing balance with their own strength. Therefore, the vertical exoskeleton lower limb rehabilitation robot is often equipped with weight-reducing balance mechanism and medical treadmill.

Weight-reducing balance mechanism is generally composed of weight-reducing bracket, height of gravity centre adjusting mechanism, safety handrails and pneu- 
matic springs. The height of gravity centre adjusting mechanism adopts parallelogram mechanism, which can only move up and down in vertical direction. This not only satisfies the change of gravity centre in the process of rehabilitation training with exoskeleton, but also prevents hemiplegic patients from tilting left and right in horizontal direction and moving forward and backward with treadmill. The emergency stop switch can be installed on the safety handrail to ensure safety. The pneumatic spring can balance the weight of the exoskeleton and reduce the vibration of the lower limb exoskeleton and the balance support module while adapting to the change of the patients' position in the vertical direction.

Medical treadmills are responsible for driving foot movements.

\subsection{Foot Pedal Structure Design}

The biggest difference between pedal-type lower limb rehabilitation robot and exoskeleton-type is that its assistant training mechanism is pedal. They are connected with the patient's feet, and the rehabilitation training of hip, knee and ankle joint is completed on vertical plane by driving feet. The training mechanisms include the left leg and the right leg. The two sets of mechanisms are completely symmetrical. The distance between the center of the mechanisms is approximately the distance between the two hip joints of the human body, which ensures that the lower limbs are trained on the vertical plane in the course of rehabilitation. The left and right hip and knee joint change in inverse phase during the reciprocating motion of the left and right leg mechanism.

The pedals are designed to couple left and right. Because they only contact with the patient's feet, they need to be fixed in order to avoid the side-shifting and turning of the patient's legs. Usually, the exoskeleton protective mechanism is used.

\section{Control System Design of Lower Limb Rehabilitation Robot}

The design of the general control scheme of rehabilitation robot focuses on two aspects: one is to ensure the high safety of the robot from the design of the control system; the other is to meet the requirements of rehabilitation training from the clinical point of view.

The impedance control method is used in the design of the control system, which allows the deviation of the trajectory when the force increases, and makes the robot to have a certain degree of flexibility. Considering the intelligent control mode, the adaptive performance of the system is increased. Sensors and other measuring elements are used to set the range of some key parameters in the training process. Once the range exceeds the pre-set range, the training will be stopped immediately. Limit switch and emergency stop button are used for multiple protection.

\subsection{Components of Control System}

The control system is mainly divided into central control system, human-computer interaction system, sensor measurement system and motion control system. The 
central control system receives instructions from the human-computer interaction system, transfers them to the motion control system, and modifies the instructions in real time according to the feedback information of the sensor measurement system.

\subsubsection{Central Control System}

The central control system is responsible for managing the whole control system and running the upper control algorithm.

There are generally two kinds of control methods for robotic systems: centralized control and hierarchical control. Centralized control means that all motor control algorithms are implemented by a single computer, characterized by small scale, high integration and high reliability, but the control program is complex and real-time is not easy to guarantee; hierarchical control means that the control algorithm is accomplished on the upper computer, while the lower computer generally uses multiple single-chip computers, each single-chip computer only controls one motor in a closed loop, and communicates data and control instructions with the upper computer through communication interface, which is characterized by good real-time performance.

\subsubsection{Human-Computer Interaction System}

Human-computer interaction system is mainly used to set working mode and set relevant parameters to show the effect of rehabilitation training. The human-computer interaction device is a touch screen, which is mainly used for physicians to check and verify the clinical rehabilitation process, including physiological parameters, muscle strength information, training intensity, speed and other indicators. Patients can also view their own rehabilitation training effect through background data, choose their own training parameters, or choose different virtual training environment in the process of rehabilitation training to increase training interest and confidence. In the graphical human-computer interaction interface, real-time training status information such as joint angle, speed and moment collected synchronously from the sensor system in the training process can be displayed, so that patients or physicians can timely and intuitively understand the patient's motion status, motion characteristics, motor function level and rehabilitation level.

\subsubsection{Sensor Measurement System}

In order to accurately grasp the recovery of lower limbs during rehabilitation, the robot and lower limbs are equipped with corresponding position sensors, angle sensors and force sensors or with a motion capture system equipped with a camera, so that therapists and patients can see the real-time changes of training parameters during rehabilitation on the monitor and provide feedback data for the central control system.

\subsubsection{Motion Control System}

Considering that the rehabilitation robot is in a low-speed state in the training 
process, the load of the robot is a patient with lower limb motor dysfunction, and there will be great load changes in the training process. Therefore, the system is driven by a torque motor and equipped with a servo motor controller.

\subsection{Control Strategy}

In order to train the lower limb joints and muscle strength, the rehabilitation robot needs to drive the lower limbs to complete the pre-planned training trajectory at a certain speed. The majority of patients with lower limb motor dysfunction will experience three stages in the rehabilitation process: paralysis, spasm and recovery. Rehabilitation training needs to adopt different control strategies to achieve the required training mode according to the rehabilitation purpose of different stages.

According to the training needs of patients in different rehabilitation stages, the robot control system should provide passive training, assistant training and active training mode. For patients with severe illness, it is suitable to adopt passive training mode, follow the principle of clinical rehabilitation from proximal joints (hip joints) to distal joints (ankle joints). Firstly, joint separation training is carried out, and then coordinated training is carried out, so that all joints can maintain the maximum range of motion. For patients with mild illness, the assistant training mode or active training mode can be used to increase the muscle strength and endurance of lower limbs and strengthen the coordinated movement of the joints.

\subsubsection{Passive Training}

When implementing passive control, the trajectory of the end position of the robot should be planned according to the training requirement of the limb joint. Then the desired trajectory of the driving joint should be solved according to the inverse kinematics analysis. The trajectory tracking control can be carried out through the position controller to achieve the desired trajectory tracking [8] [9] [10].

In the control system, each motor is an independent closed-loop servo system. Considering the low speed of rehabilitation training, the load will change. The most basic performance index of trajectory control is the stability of the system. At the same time, the torque motor has the characteristics of low speed and small torque fluctuation, so it can control the position of the motor closed-loop servo system in a single closed-loop way.

With the prolongation of the course of disease, muscle strength changes gradually, and abnormal movement patterns will appear in the rehabilitation process, which will lead to great changes in the contact force between human and robot, and make the actual training trajectory deviate from the pre-planned trajectory. At this time, if the trajectory control method is used only, the contact stiffness between human and robot will increase due to the position control method, which will easily lead to secondary damage. In the course of training, we should not only prevent the occurrence of accidents such as muscle strain 
and joint injury, but also ensure the safety and comfort of patients' training.

\subsubsection{Assistant Training}

With the extension of rehabilitation training time, the muscle strength of lower limbs gradually increased. When the lower limbs have certain initiative, but do not have enough muscle strength to complete the active training, the assistant training mode can be used for the lower limbs. When the lower limb exerts an active force on the robot, the plantar contact force is detected by sensors, the force variation is obtained and judged, and the patient's active motion trend is analysed. Given the desired current signal of the control system according to the active movement trend, the motor works in the current loop control mode. It realizes the control of the motor output torque, and completes the rehabilitation training of the lower limbs and the motor providing part of the force to assist the patients.

\subsubsection{Active Training}

The control system works in the active control mode, that is, using the lower limbs to exert driving force on the robot, detecting the contact force between human and robot by sensors, and converting the stiffness coefficient $\mathrm{K}$ into the input signal of the control system, so that the robot can realize the active training mode needed in clinic.

\section{Research Progress of Lower Limb Rehabilitation Robots}

\subsection{Harbin Engineering University}

Harbin Engineering University is an early and mature institution for the study of lower limb rehabilitation robots in mainland China. Its main research is the horizontal foot pedal lower limb rehabilitation robot (see Figure 1, Figure 2) [11] [12] [13] [14].

Compared with the exoskeleton lower limb rehabilitation robot, this robot

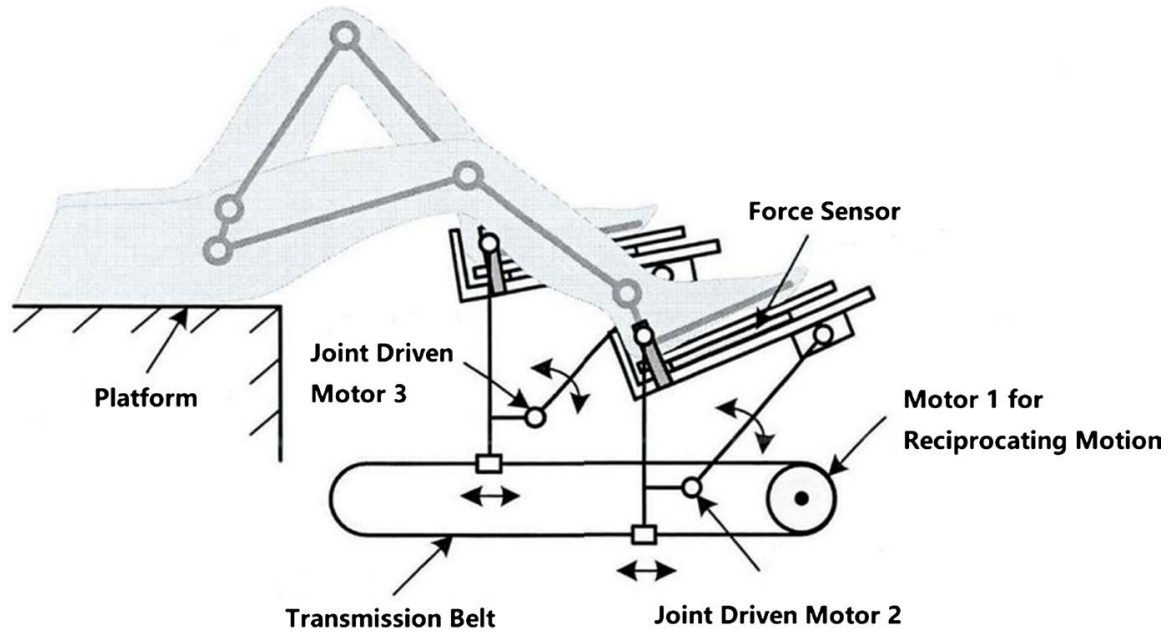

Figure 1. Principle diagram of horizontal foot pedal lower limb rehabilitation robot of Harbin Engineering University. 
provides an additional active-passive training mode. Impedance control and fuzzy control are adopted for the possible abnormal movement and spasm of the lower limbs, which greatly reduces the possibility of secondary injury. However, the force sensor and position sensor are located at the foot pedal, which cannot effectively monitor the motion state of lower limb muscles in real time. At the same time, although the horizontal platform provides a support structure for patients, it cannot really restore the human walking environment. Therefore, the robot has a certain effect on restoring muscle strength of lower limbs, but it cannot complete gait training.

\subsection{Yanshan University}

Yanshan University also belongs to mature domestic institution for the study of lower limb rehabilitation robots is the sitting and horizontal exoskeleton lower limb rehabilitation robot (see Figure 3) [15]-[21]. Its shape is similar to the traditional massage machine, and its structure is compact. The control system is located in the box under the seat. The robot has a good human-computer interaction

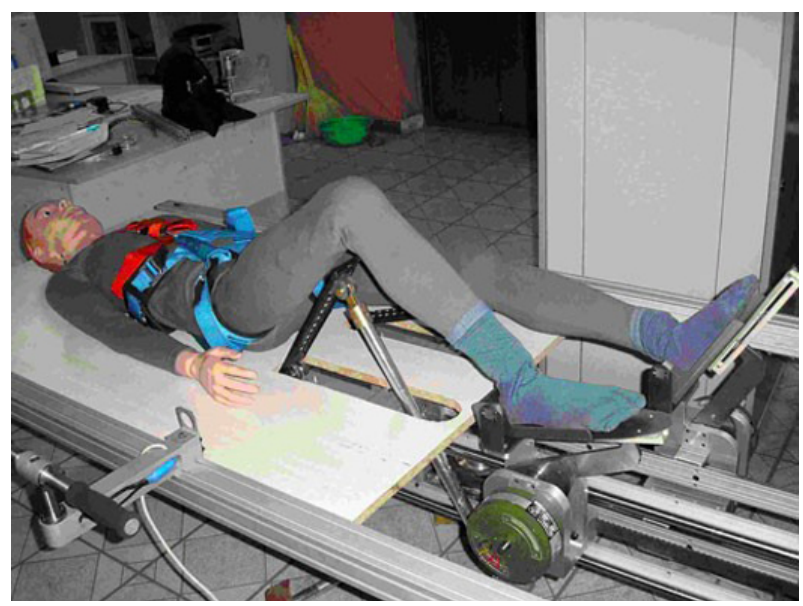

Figure 2. Horizontal foot pedal lower limb rehabilitation robot of Harbin Engineering University.

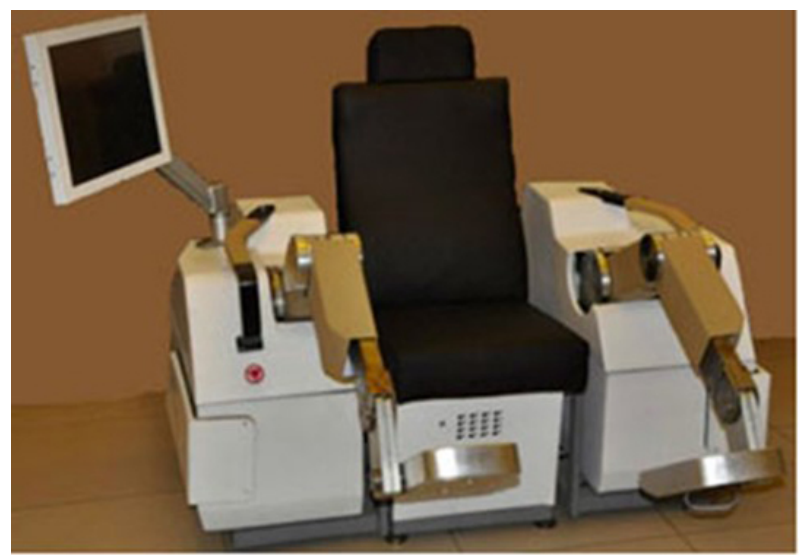

Figure 3. Experimental prototype of sitting and horizontal exoskeleton lower limb rehabilitation robot of Yanshan University. 
platform. Its advantage lies in the use of EMG signal acquisition card, which can accurately monitor the motion intention of leg muscle groups in real time. The effect of muscle strength recovery is obvious, but the effect of gait training is not significant.

\subsection{Tsinghua University}

Tsinghua University, as the earliest Institute in mainland China to study lower limb rehabilitation robot, has expanded its research direction from the original horizontal foot pedal lower limb rehabilitation robot similar to bicycle principle to the exoskeleton lower limb rehabilitation robot with weight-reducing balance frame (see Figure 4). It is similar to Lokomat of Hocoma (Switzerland), and has obvious effect on patients' gait recovery training. But its disadvantage is that in order to solve the problem that the position control algorithm lags behind the actual position, the position control is only used in the swing phase, which will cause slippage between the sole of the foot and the conveyor belt of the medical runway, and the frequent switch of the motor will lead to vibration [22] [23] [24] [25].

\subsection{Shanghai Jiao Tong University}

Shanghai Jiao Tong University is also representative in the field of lower limb rehabilitation robot research. Figure 5 is its vertical exoskeleton lower limb rehabilitation robot [26] [27] [28]. The robot draws lessons from eLegs of Berkeley Bionics Technology Company.

The robot gets rid of the restriction of the site, uses crutches to support most of the body's weight. The crutches take on the function of controlling the driving mechanism. However, it lacks a sensing and measuring system, and can only be used as an assistant robot. Its rehabilitation effect is poor.

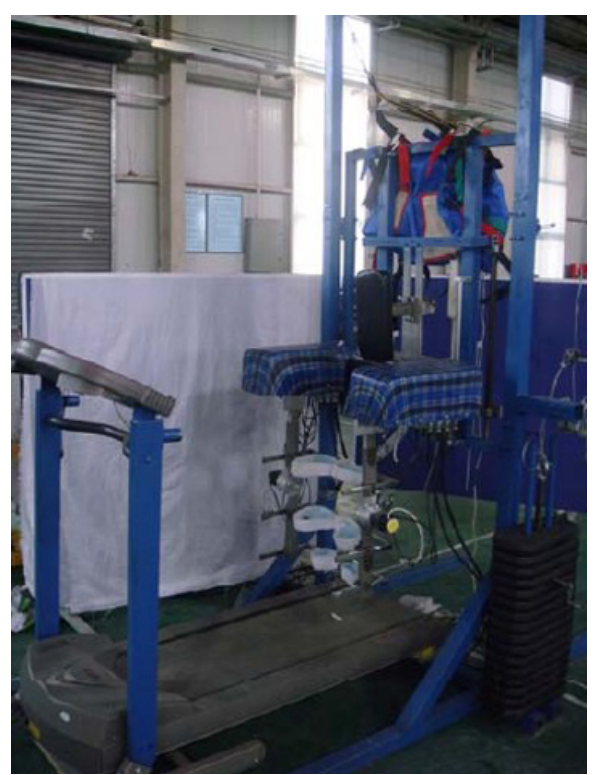

Figure 4. Vertical exoskeleton lower limb rehabilitation robot of Tsinghua University. 


\subsection{Harbin Institute of Technology}

Figure 6 is Vertical exoskeleton lower limb rehabilitation robot of Harbin Institute of Technology. The robot also draws lessons from Lokomat. Its advantage is that it replaces traditional PD control and adopts fuzzy adaptive control [29] [30] to improve flexibility, and adds two degrees of freedom to the sole of the foot to achieve heel landing first and toe landing later. The disadvantage lies in

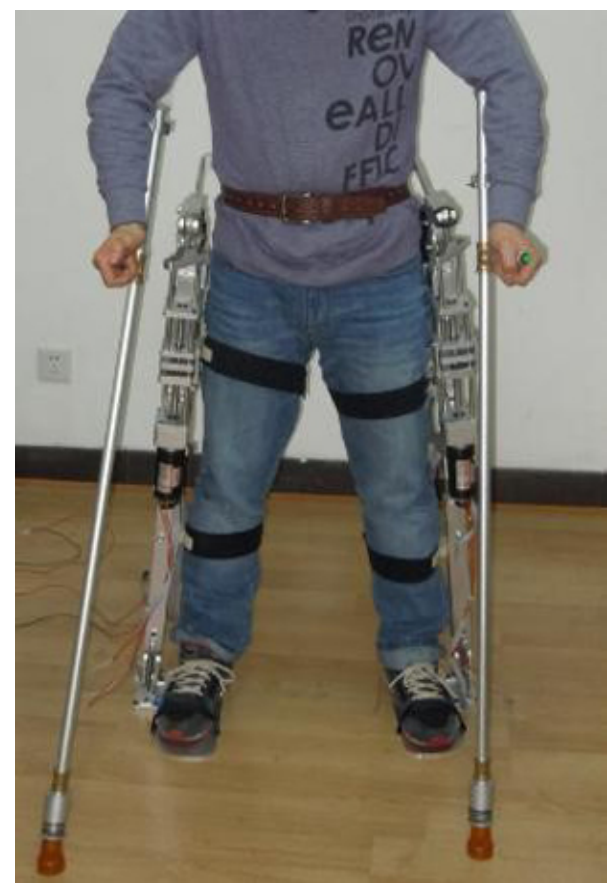

Figure 5. Vertical exoskeleton lower limb rehabilitation robot of Shanghai Jiao Tong University.

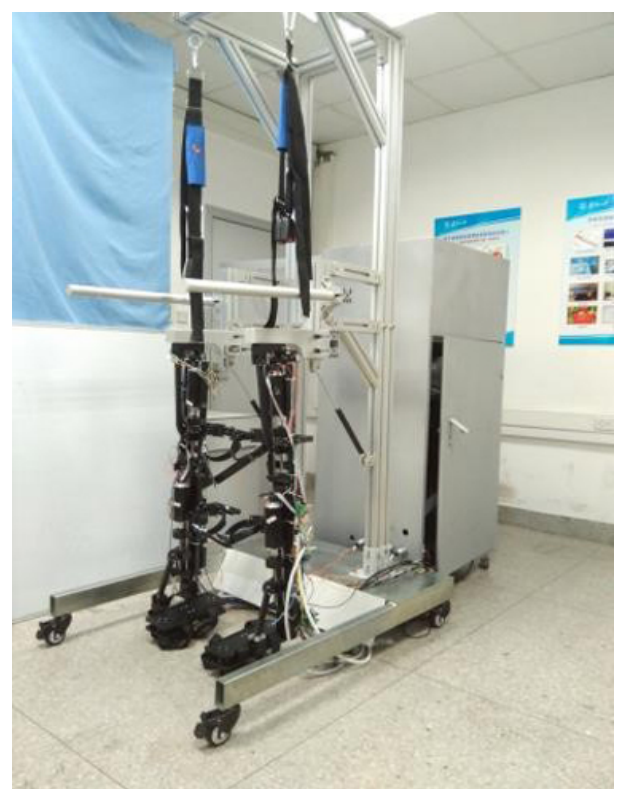

Figure 6. Vertical exoskeleton lower limb rehabilitation robot of Harbin Institute of Technology. 
the lack of conveyor belts and the failure to simulate human normal gait [31] [32].

\section{Conclusions}

In this paper, some research results of lower limb rehabilitation robots in mainland China are summarized.

1) At present, most of the domestic researches on lower limb rehabilitation robots are lower limb rehabilitation training robots, while the design requirements of lower limb rehabilitation assistant robots are more than those of the former and lower limb rehabilitation assistant robots are not the main requirements, so the research content is less. The structural design direction of domestic lower limb rehabilitation training robots is mainly horizontal foot pedal lower limb rehabilitation robots and weight-reducing exoskeleton lower limb rehabilitation robots.

2) In the design of control system, the central control system adopts hierarchical control. Force sensor, position sensor and angle sensor are commonly used in sensor measurement system. The sole pedal type is equipped with position sensor and force sensor at the pedal. The exoskeleton type is equipped with angle sensors at each joint and force sensors at leg muscles. In the design of motion control system, there are two ways: screw-nut drive and direct drive. Because the servo motor does not need higher speed in passive control and needs larger torque, most of them choose screw-nut drive. However, this method needs complex conversion between motor rotation angle and leg rotation angle, which will lead to larger control error, and this structure does not have reverse drive. In terms of control strategy, the general lower limb rehabilitation robots only include passive, active and assistant modes.

3) Through the analysis of the rehabilitation effect of horizontal foot pedal lower limb rehabilitation robot and weight-reducing exoskeleton lower limb rehabilitation robot, it is found that although the former is easy to achieve in design, it is inferior to the latter in gait training.

In summary, at present, the weight-reducing exoskeleton lower limb rehabilitation robot is more mature in domestic design and has better rehabilitation effect. In future research, researchers will focus on finding new materials and optimizing structures to reduce the weight of rehabilitation robots. On the other hand, the feedback accuracy optimization of sensor measurement system and the algorithm optimization of motion control system are also hot issues to be solved urgently by researchers.

\section{Conflicts of Interest}

There is no conflict of interest regarding the publication of this paper.

\section{References}

[1] Li, H., Yao, H.H. and Liu, L.H. (2003) Effects of Muscle Strengthening Exercise on 
Gait of Stroke Patients and the Relationship between the Evaluation of Gait Analysis and Functional Assessment. Chinese Journal of Physical Medicine and Rehabilitation, 25, 34-36.

[2] Hu, Y., et al. (2007) Standardized Tertiary Rehabilitation on the Comprehensive Functions of Cerebral Strokes Patients with Hemiplegia. Chinese Journal of Rehabilitation Medicine, 22, 3-7.

[3] Xie, C.Z., Xu, G.L. and Liu, X.F. (2009) Advance in Early Rehabilitation after Stroke. Chinese Journal of Rehabilitation Theory and Practice, 15, 908-912.

[4] Lunenburger, L., Colombo, G. and Riener, R. (2005) Clinical Assessments Performed during Robotic Rehabilitation by the Gait Training Robot Lokomat. IEEE Proceedings of the 9th International Conference on Rehabilitation Robotics, Chicago, 28 June-1 July 2005, 345-358. https://doi.org/10.1109/ICORR.2005.1501116

[5] Lunenburger, L., Gery, C. and Robert, R. (2004) Biofeedback in Gait Training with the Robotic Orthosis Lokomat. The 26th Annual International Conference of the IEEE Engineering in Medicine and Biology Society, San Francisco, 1-5 September, 2004, 4888-4891. https://doi.org/10.1109/IEMBS.2004.1404352

[6] Hayashi, T., Kawamoto, H. and Sankai, Y. (2005) Control Method of Robot Suit HAL Working as Operator's Muscle Using Biological and Dynamical Information. 2005 IEEE/RSJ International Conference on Intelligence Robots and Systems, Edmonton, 2-6 August 2005, 3455-3460. https://doi.org/10.1109/IROS.2005.1545505

[7] Kawamoto, H. and Sankai, Y. (2002) Power Assist System HAL-3 for Gait Disorder Person. Proc. of International Conference on Computers Helping People with Special Needs, Linz, 15-20 July 2002, 196-203. https://doi.org/10.1007/3-540-45491-8_43

[8] Cheng, F., et al. (2008) Gait Programming Algorithm for the Gait Training Robot Used in PBWST. Chinese Journal of Rehabilitation Medicine, 23, 916-918.

[9] Wang, A.M., Jia, X.H., Wang R.C., et al. (2008) Preliminary Research on Motion Planning of Partial Body Weight Support Treadmill Training Rehabilitation Robot. The 3rd Beijing International Forum on Rehabilitation, Beijing, 23-28 October 2008, 407-409.

[10] Cheng, F., Wang, R.C., Jia, X.H., et al. (2008) Advance in Partial Body Weight Support Treadmill Training Rehabilitation Robot. Chinese Journal of Rehabilitation Medicine, 23, 366-368.

[11] Sun, H., Zhang, L. and Wang, L. (2010) Dynamics Modeling and Control of Horizontal Lower Limbs Rehabilitation Robot. Chinese High Technology Letters, 20, 733-738.

[12] Qian, Z.M., et al. (2010) Kinematics Analysis and Simulation of Horizontal Lower Limbs Rehabilitative Robot. Journal of System Simulation, 22, 2001-2005.

[13] Sun, H., Zhang, L. and Li, C. (2009) Dynamic Analysis of Horizontal Lower Limbs Rehabilitation Robot. 2009 IEEE International Conference on Intelligent Computing and Intelligent Systems, Shanghai, 20-22 November 2009, 656-660. https://doi.org/10.1109/ICICISYS.2009.5358301

[14] Zhang, L., Sun, H. and Li, C. (2010) Experiment Study of Impedance Control on Horizontal Lower Limbs Rehabilitation Robot. The 2010 IEEE International Conference on Information and Automation, Harbin, 20-23 June 2010, 1421-1425.

[15] Shi, X., Ren, L., Liao, Z., Zhu, J. and Wang, H. (2017) Design \& Analysis of the Mechanical System for a Spacial 4-DOF Series-Parallel Hybrid Lower Limb Rehabilitation Robot. Journal of Mechanical Engineering, 53, 48-54.

https://doi.org/10.3901/JME.2017.13.048 
[16] Shi, X.H., Lu, H., Liao, Z.Y., et al. (2018) Active Training of Lower Limb Rehabilitation Robot Based on sEMG. Science Technology and Engineering, 18, 61-66.

[17] Zhao, X.L., Lin, M.S., Li, Q., et al. (2016) Teaching and Training of the Lower Limb Rehabilitation Robot Based on Accelerometer. Chinese Journal of Sensors and Actuators, 29, 1596-1601.

[18] Shi, X. (2014) Design and Dynamic Analysis of an Exoskeleton Lower Limbs Rehabilitation Robot. Journal of Mechanical Engineering, 50, 41-48. https://doi.org/10.3901/JME.2014.03.041

[19] Wang, H., Shi, X. and Liu, H. (2011) Design, Kinematics, Simulation, and Experiment for a Lower-Limb Rehabilitation Robot. Proceedings of the Institution of Mechanical Engineers, Part I: Journal of Systems and Control Engineering, 225, 860-872. https://doi.org/10.1177/0959651811408978

[20] Shi, X.H., et al. (2012) Design and Analysis of a Lower Limb Rehabilitation Robot. Advanced Materials Research, 490-495, 2236-2240. https://doi.org/10.4028/www.scientific.net/AMR.490-495.2236

[21] Wang, H., Liu, H., Shi, X. and Hou, Z. (2009) Design and Kinematics of a Lower Limb Rehabilitation Robot. 2nd International Conference on Biomedical Engineering and Informatics, Tianjin, 17-19 October 2009, 1174-1177.

[22] Chen, K., Liu, Q.D., Wang, R.C., et al. (2011) Development of a Body Weight-Support Gait Training Robot. Chinese Journal of Rehabilitation Medicine, 26, 847-851.

[23] Liu, Q.D., Chen, K., Wang, R.C., et al. (2010) Development of Body Weight-Support Gait Training Robot. The 7 th National Symposium on Rehabilitation Medical Engineering and Rehabilitation Engineering, Beijing, 22-24 May 2010, 81-84.

[24] Zhang, T.Y., Liu, Q.D., Wang, R.C., et al. (2010) The Standardization of Body Weight Support Intelligent Instrument for Gait Training. The 7 th National Symposium on Rehabilitation Medical Engineering and Rehabilitation Engineering, Beijing, 22-24 May 2010, 299-302.

[25] Wang, A.M., Wang, R.C., Chen, K., et al. (2008) Research Development of Exoskeleton Robot for Assistive Walking. The 3rd Beijing International Forum on Rehabilitation, Beijing, 23-28 October 2008, 567-569.

[26] Rao, L.J., Xie, L. and Zhu, X.B. (2012) Research and Design on Lower Exoskeleton Rehabilitation Robot. Machine Design and Research, 28, 24-28.

[27] Zhu, X.B., Xie, L., Xv, D., et al. (2013) Numerical Research of Walking Motion on a Lower Limb Exoskeleton. China Digital Medicine, 8, 27-29.

[28] Zhu, X.B., Xie, L. and Shen X.L. (2012) Passive Control Scheme for a Lower Extremity Rehabilitation Exoskeleton. 1st International Conference of Rehabilitation Medical Engineering, Shanghai, 17-18 May 2012, 17-18.

[29] Li, F., Wu, Z.Z. and Qian, J.W. (2014) Trajectory Adaptation Control for Lower Extremity Rehabilitation Robot. Chinese Journal of Scientific Instrument, 35, 2027-2036.

[30] Xu, G.Z., Song, A.G. and Li, H.J. (2009) Fuzzy-Based Adaptive Impedance Control for Upper-Limb Rehabilitation Robot. Journal of Southeast University (Natural Science Edition), 39, 156-160.

[31] Zhou, H., Sun, L., Li, J., Li, W., Cai, X. and Lu, L. (2015) Adaptive Gait Trajectory Based on Iterative Learning Control for Lower Extremity Rehabilitation Exoskeleton. 18 th IEEE International Conference on Climbing and Walking Robots, Hangzhou, 6-9 September 2015, 43-50. 
[32] Zhou, H.T. (2015) Research on Mechanism Design and Control Strategy of the Lower Extremity Rehabilitation Exoskeleton. Ph.D. Dissertation, Harbin Institute of Technology, Harbin. 